\title{
Calculable corrections to brane black hole decay: The scalar case
}

\author{
Panagiota Kanti and John March-Russell \\ Theory Division, CERN, CH-1211 Geneva 23, Switzerland
}

(Received 12 April 2002; published 18 July 2002)

\begin{abstract}
In the context of brane-world theories, the production cross section for black holes may be greatly enhanced. Such black holes can in principle lead to detectable signals via their Hawking evaporation to brane-localized modes. We calculate, in the semiclassical approximation, the leading corrections to the energy spectrum (the greybody factors) for decay into scalar fields, as a function of the number of toroidally compactified extra dimensions, and partial wave number.
\end{abstract}

DOI: 10.1103/PhysRevD.66.024023

PACS number(s): 04.70.Dy, 04.50.+h, 11.10.Kk

\section{INTRODUCTION}

It is a remarkable fact that in brane-world theories the true scale $M_{*}$ of quantum gravity may be substantially lower than the traditional Planck scale $M_{\mathrm{pl}}$, possibly approaching the $\mathrm{TeV}$ scale, and this radical departure from the standard picture is not excluded [1] (for earlier works on brane theories, see [2]). This observation has naturally excited a large amount of activity investigating both the structure of these theories and their experimental signals [3]. One of the most striking consequences of lowering the Planck scale to the $\mathrm{TeV}$ region is that the properties of small black holes are substantially altered [4]. A black hole of given mass $M$ is now much lighter, larger, and colder than a usual black hole of the same mass, provided only that the Schwarschild radius $r_{H}$ of the black hole $(\mathrm{BH})$ is smaller than the size of the extra dimensions $r_{H}<R$. In this limit, the black hole is well described as a $(4+n)$-dimensional black hole centered on the brane, but extending out into the $n$ extra dimensions. The horizon radius $r_{H}$ of such a black hole is [4,5]

$$
r_{H}=\frac{1}{M_{*}}\left(\frac{M}{M_{*}}\right)^{1 /(n+1)}\left(\frac{8 \Gamma[(n+3) / 2]}{(n+2) \pi^{(n+1) / 2}}\right)^{1 /(n+1)} \text {. }
$$

[Note that, following common practice, we work in the approximation that the brane tension itself does not strongly perturb the $(4+n)$-dimensional black hole solutions.]

In particular, it is likely (though not proven) that black holes are much easier to produce, with production cross section at parton-parton center-of-mass (c.m.) energies $\sqrt{s}$ close to the geometrical cross section of a black hole of mass $M$ $=\sqrt{s}[6,7]$ (for supporting evidence see, e.g., [8]; for claims to the contrary, see [9]):

$$
\sigma_{\text {prod }}(s) \simeq \pi r_{H}^{2}=\frac{1}{M_{*}^{2}}\left(\frac{M}{M_{*}}\left\{\frac{8 \Gamma\left(\frac{n+3}{2}\right)}{n+2}\right\}\right)^{2 /(n+1)} .
$$

If this is the case, then for $M_{*} \sim \mathcal{O}(\mathrm{TeV})$ there are striking consequences for the high-energy interactions of cosmic rays [10], and, moreover, the CERN Large Hadron Collider (LHC) will become a "black hole factory" $[6,7,11]$.

After such black holes are produced, they decay by the emission of Hawking radiation. It is expected that black holes produced by a collision on our brane, e.g., $p \bar{p} \rightarrow \mathrm{BH}$ $+X$ at a hadron collider, will decay mostly to particles on our brane [12], and thus will be indirectly observable via this characteristic Hawking radiation. This radiation is usually described as "thermal" in character with a temperature

$$
T_{\mathrm{BH}}=\frac{(1+n)}{4 \pi} \frac{1}{r_{H}}
$$

(in $G_{N}=k_{B}=c=\hbar=1$ units). However, because of the nontrivial metric in the region exterior to the horizon, there exists an effective potential barrier in this exterior region. This potential barrier backscatters a part of the outgoing radiation back into the black hole, the amount depending on the energy of the radiation. Thus the original blackbody radiation is modified by a frequency-dependent filtering function, $\sigma(\omega)$, caused by the gravitational potential of the black hole. The function, $\sigma(\omega)$, is known as the "greybody factor." The black hole differential decay rate into particles of energy $\omega$ is then given by the Hawking formula [13]

$$
\frac{d E(\omega)}{d t}=\sum_{l, b} \sigma_{l, b}(\omega) \frac{\omega}{\exp \left(\omega / T_{\mathrm{BH}}\right) \mp 1} \frac{d^{n+3} k}{(2 \pi)^{n+3}},
$$

where $l$ labels the angular momentum quantum number, $b$ labels any other quantum numbers of the emitted particle, as well as the particle type, and in the phase-space integral $|k|$ $=\omega$ for a massless particle. ${ }^{1}$

Greybody factors are important theoretically because they encode information on the near-horizon structure of black holes. Indeed one of the most exciting features of $\mathrm{BH}$ production at the LHC would be the opportunity to investigate Hawking emission in detail for clues as to how the infamous apparent violation of the laws of quantum mechanics by black hole evaporation (the "information paradox" of black holes [14]) is resolved-if it is, that is.

Greybody factors can be important experimentally because they modify the spectrum in the region where most

\footnotetext{
${ }^{1}$ An alternate form for the decay rate that is sometimes useful in the massless particle case involves the absorption probability $|\mathcal{A}(\omega)|^{2}$ whose relation to $\sigma(\omega)$ is given in Eq. (13): $d E(\omega) / d t$ $=\Sigma_{l, m, b}|\mathcal{A}(\omega)|^{2} \omega d \omega /\left\{2 \pi\left[\exp \left(\omega / T_{\mathrm{BH}}\right) \mp 1\right]\right\}$. Here $m$ is the azimuthal quantum number.
} 
particles are produced, thus altering the characteristic spectrum by which we hope to identify a "BH event." In particular, the functional dependence of $\sigma_{b}(\omega)$ on the energy $\omega$ depends on the spin of the emitted particle, and on whether it is brane-localized or free to propagate in the bulk of the extra dimensions.

One can compute the greybody factor by first computing the absorption cross section for the appropriate type of particle incident on the background metric that describes the brane black hole. This is because the greybody factor in the Hawking formula for the emission rate of a given type of outgoing particle, $b$, at energy $\omega$ equals the absorption cross section $\sigma_{b \text {,abs }}(\omega)$ for a particle of type $b$ incoming at energy $\omega$. In fact, it is this property which implies the greybody factors do not invalidate the thermal nature of the black hole. Since the outgoing transmission and incoming absorption coefficients are equal to one another, equilibrium still occurs if the black hole is placed in a heat bath.

The semiclassical calculation of Hawking emission is only reliable when the energy of the emitted particle is small compared to the black hole mass $\omega \ll M$, since only in this case is it correct to neglect the backreaction of the metric during the emission process. This in turn requires that the Hawking temperature $T_{\mathrm{BH}} \ll M$, which is equivalent to demanding that the black hole mass $M \gg M_{*}$, as can be seen from Eqs. (3) and (1). Inevitably this condition breaks down during the final stages of the decay process, but for those black holes of initial mass larger than $M_{*}$ most of the evaporation process is well described by the semiclassical calculation.

In this paper, we calculate the greybody factor for the simplest case of scalar particles, both free to propagate in the bulk and localized on the brane (e.g., the Higgs boson). Section II contains a discussion of the metric used in calculating the greybody factors and the quality of the approximations used. To orient the reader through our analysis, Sec. III presents the calculation of bulk scalar emission in $(4+n)$ dimensions, in the simple case of $S$-wave emission. Section IV performs the calculation of the greybody factor for bulk scalar emission for arbitrary partial wave. The primary result of this section, the general formula for the greybody factor, is given in Eq. (37). Section V contains the calculation of brane-localized scalar emission, where the brane is embedded in a $(4+n)$-dimensional bulk. The primary results of this section are given in Eqs. (47) and (48). We conclude in Sec. VI. For ease of use, the results of our calculations for the greybody factors, for the most important $(l=0,1,2)$ angular momentum modes, and for the $n=2,4,6$ extra dimensions are collected in Tables I and II.

\section{BLACK-HOLE METRICS AND GREYBODY FACTORS}

Let $V=(2 \pi R)^{n}$ be the volume of the $n$ extra dimensions, which are here taken to be of common radius $R$. Then Gauss' law relates the four-dimensional (4D) Planck mass $M_{\mathrm{pl}}$ to the new fundamental scale of gravity by $M_{\mathrm{pl}}^{2}=V_{n} M_{*}^{2+n}$. A black hole of mass $M \ll M_{\mathrm{pl}}\left(M_{\mathrm{pl}} / M_{*}\right)^{(2+n) / n} \sim 10^{15+32 / n} \mathrm{GeV}$ is, for distances $r \ll R$, well approximated [4] by the $(4+n)$-dimensional Schwarzschild black hole with a line element

$$
d s^{2}=-h(r) d t^{2}+h(r)^{-1} d r^{2}+r^{2} d \Omega_{2+n}^{2},
$$

where

$$
h(r)=1-\left(\frac{r_{H}}{r}\right)^{n+1}
$$

with the horizon radius, $r_{H}$, given in Eq. (1). In Eq. (5), the angular part is

$$
d \Omega_{2+n}^{2}=d \theta_{n+1}^{2}+\sin ^{2} \theta_{n+1}\left\{d \theta_{n}^{2}+\sin ^{2} \theta_{n}\left[\cdots+\sin ^{2} \theta_{2}\left(d \theta_{1}^{2}+\sin ^{2} \theta_{1} d \varphi^{2}\right) \cdots\right]\right\},
$$

with $0<\varphi<2 \pi$ and $0<\theta_{i}<\pi$ for $i=1, \ldots, n+1$. However, because of the compactification of the extra dimensions, the metric for this black hole at distances $r \gg R$ goes over to that of the usual four-dimensional Schwarzschild solution

$$
d s^{2}=-\left(1-\frac{2 M}{M_{\mathrm{Pl}}^{2} r}\right) d t^{2}+\left(1-\frac{2 M}{M_{\mathrm{Pl}}^{2} r}\right)^{-1} d r^{2}+r^{2} d \Omega_{2}^{2}
$$

The matching of the two expressions Eqs. (5) and (8) at $r \simeq R$ is of course just an approximation to the exact metric for such a black hole. An exact expression is not necessary to derive the form of the greybody factor to a very good approximation for the energies of interest.

To understand this, consider the case in which a scalar field propagates in the full $(4+n)$-dimensional Schwarz- schild black hole background. The scalar wave equation $g^{I J} \phi_{, I ; J}=0$ in this background is separable if we make the ansatz

$$
\phi\left(t, r, \theta_{i}, \varphi\right)=e^{-i \omega t} R_{\omega l}(r) \widetilde{Y}_{l}(\Omega),
$$

where $\tilde{Y}_{l}(\Omega)$ is the $(3+n)$-spatial-dimensional generalization of the usual spherical harmonic functions depending on the angular coordinates [15]. Upon substituting this ansatz, the scalar wave equation implies a second-order differential equation for the radial wave function $R_{\omega l}(r)$ :

$$
\frac{h(r)}{r^{n+2}} \frac{d}{d r}\left[h(r) r^{n+2} \frac{d R}{d r}\right]+\left[\omega^{2}-\frac{h(r)}{r^{2}} l(l+n+1)\right] R=0 .
$$


The greybody factor for $\mathrm{BH}$ decay into scalars will be found from the solutions to this equation.

We can transform this equation to a more convenient form by defining a new ("tortoise") radial coordinate $y$ by

$$
y=\frac{\ln h(r)}{r_{H}^{n+1}(n+1)} \Rightarrow \frac{d y}{d r}=\frac{1}{h(r) r^{n+2}}
$$

in terms of which the radial equation becomes

$$
\left(\frac{d^{2}}{d y^{2}}+r^{2 n+4}\left[\omega^{2}-\frac{l(l+n+1) h(r)}{r^{2}}\right]\right) R(y)=0 .
$$

Since the coordinate position $r_{H}$ of the horizon is defined by the (largest) solution to $h\left(r_{H}\right)=0$, the horizon in terms of $y$ is at $y \rightarrow-\infty$, as can been seen from Eq. (11). Equation (12) is analogous to the Schrödinger equation for a particle in an effective potential.

Nontrivial backscattering occurs in this metric when the second of the two terms in the square brackets in Eq. (12) is comparable to or larger than the first. For black holes at the LHC, the typical energies of emitted radiation we are interested in range from $\omega_{\max } \sim T_{\mathrm{BH}}=(1+n) / 4 \pi r_{H}$ $\sim$ (few) $100 \mathrm{GeV}$ to a minimum of $\sim$ (few) $\mathrm{GeV}$ (this minimum might be set by the energy threshold of the detectorsthe precise value will not matter). For the range of parameters $n$ and $M_{*}$ of interest, this gives a range of $\omega$ from $\omega_{\max } \leqslant 1 / 2 r_{H}$ to $\omega_{\min } \geq 1 / 200 r_{H}$. Thus, inspecting the potential terms of Eq. (12), we see that the potential is only large enough to lead to backscattering over the range of distances $r$ from $O(1) r_{H}$ to $O(100) r_{H}$.

A similar analysis can be performed for the 4D asymptotic metric of Eq. (8). In this case, one finds that significant backscattering of the quanta of interest would only occur when distances were of order $r \sim M / M_{\mathrm{Pl}}^{2} \ll r_{H}$ $\ll R$. But at such distances, the $4 \mathrm{D}$ asymptotic form of the metric is not applicable, being replaced by the $(4+n)$-dimensional metric used above. Instead, what happens is that the changeover from the $(4+n)$-dimensional to the $4 \mathrm{D}$ regime, at distances of order $r \sim R$, only significantly backscatters quanta of energy $10^{-3} \mathrm{eV}$ or less. In other words, the backscattering due to the effect of the compactification of the extra dimensions only affects very low energy quanta which are experimentally irrelevant. Even more so this applies to the change in backscattering at distances $r$ $\sim R$ due to the difference between the approximate form of the black hole metric, Eqs. (5) and (8), and the exact black hole solution.

Overall, the lesson is that up to corrections that only apply for very low energy, the greybody factors for brane black holes may be calculated using purely the $(4+n)$-dimensional regime of the black hole metric. In the remainder of this paper we will follow this procedure. Thus our task is now to return to the radial equation Eq. (10) or Eq. (12) and compute the greybody factor.

In particular, via the equivalence of $\sigma(\omega)$ to the absorption cross section, the greybody factor can be computed by first evaluating the absorption probability, $|\mathcal{A}(\omega)|^{2}$, from the ratio of the in-going flux at the future horizon to the incoming flux from past infinity (with the boundary condition that there is no outgoing flux at the horizon), and then using the generalized $(4+n)$-dimensional optical theorem relation [16]

$$
\sigma_{l}(\omega)=\frac{2^{n} \pi^{(n+1) / 2} \Gamma[(n+1) / 2]}{n ! \omega^{n+2}} \frac{(2 l+n+1)(l+n) !}{l !}|\mathcal{A}|^{2}
$$

between the absorption cross section $\sigma_{l}(\omega)$ and the absorption probability $|\mathcal{A}|^{2}$ for the $l$ th partial wave. This formula includes a summation over the multiplicity of individual "azimuthal" components for each given partial wave $l$ [i.e., $2 l+1$ in three spatial dimensions, $(l+1)^{2}$ in four spatial dimensions, etc.]. A simple summation is appropriate because we deal with a nonrotating black hole.

The radial equation, however, is not in general exactly soluble, therefore we will employ an approximation method based on splitting the radial domain into "near-horizon" $(\mathrm{NH})$ and "far-field" (FF) regions. The solutions (satisfying appropriate boundary conditions) in these two regions will be computed and then matched in a transition region to find the complete solution. This procedure leads to an expression for $\left|\mathcal{A}_{l}(\omega)\right|^{2}$ correct in leading order in an expansion in the dimensionless quantity $\omega r_{H}$.

\section{BULK SCALAR EMISSION: $S$-WAVE EXAMPLE}

Because the radius and temperature of the $(4+n)$-dimensional black hole are always comparable, Eq. (3), the dominant scalar decay mode is that into the $S$ wave, $l=0$. We will first solve the problem in this case, which will also serve as an illuminating example for the full case studied in Sec. IV and V.

We will first compute the solution in the "near-horizon" region. The radial equation for the $S$ wave, in terms of the $y$ coordinate defined in Eq. (11), becomes

$$
\left(\frac{d^{2}}{d y^{2}}+\omega^{2} r^{2(n+2)}\right) R(y)=0 .
$$

By expanding close to the horizon $r=r_{H}+\delta r\left(\delta r \ll r_{H}\right)$, we obtain $\delta r \simeq r_{H} \exp \left[(n+1) r_{H}^{n+1} y\right]$ as $y \rightarrow-\infty$. Thus the radial equation Eq. (14) becomes in the near-horizon limit

$$
\left(\frac{d^{2}}{d y^{2}}+\omega^{2} r_{H}^{2(n+2)}\right) R(y)=0,
$$

up to exponentially small corrections in $y$. The general nearhorizon solution is therefore

$$
R_{\mathrm{NH}}(y)=A_{+} \exp \left(i r_{H}^{n+2} \omega y\right)+A_{-} \exp \left(-i r_{H}^{n+2} \omega y\right) .
$$

In order to calculate the greybody factor, we must impose the boundary condition that near the horizon the solution is purely ingoing. Therefore, we need to set $A_{+}=0$.

We now turn to the far-field region which is defined by $r \gg r_{H}$. In this limit, $h(r) \simeq 1$ and, by setting $R(r)$ $=f(r) / r^{(n+1) / 2}$, Eq. (10) can be rewritten as 


$$
\frac{d^{2} f}{d r^{2}}+\frac{1}{r} \frac{d f}{d r}+\left[\omega^{2}-\frac{(n+1)^{2}}{4 r^{2}}\right] f=0
$$

which has the form of a Bessel differential equation [17]. The general solution for the radial function $R(r)$ is therefore given by

$$
R_{\mathrm{FF}}(r)=\frac{B_{+}}{r^{(n+1) / 2}} J_{(n+1) / 2}(\omega r)+\frac{B_{-}}{r^{(n+1) / 2}} Y_{(n+1) / 2}(\omega r),
$$

where $J_{(n+1) / 2}(\omega r)$ and $Y_{(n+1) / 2}(\omega r)$ are the Bessel functions of the first and second kind, respectively.

As we will soon see, the two coefficients $B_{+}$and $B_{-}$, and more specifically their ratio, will help us define the greybody factor. To compute this ratio, we need to match the far-field solution, Eq. (18), onto the near-horizon solution, Eq. (16), in the intermediate region. To this end, we expand the nearhorizon solution, in the regime $\omega r \ll 1$ and $r \gg r_{H}$, leading to $^{2}$

$$
\begin{aligned}
R_{\mathrm{NH}}(r) & \simeq A_{-} \exp \left\{i \frac{\omega r}{n+1}\left(\frac{r_{H}}{r}\right)^{n+2}\right\} \\
& \simeq A_{-}\left\{1+i \frac{\omega r}{n+1}\left(\frac{r_{H}}{r}\right)^{n+2}\right\} .
\end{aligned}
$$

We also expand the far-field solution, Eq. (18), in the same regime $\omega r \ll 1$, which gives

$R_{\mathrm{FF}}(r) \simeq \frac{B_{+}}{\Gamma\left(\frac{n+3}{2}\right)}\left(\frac{\omega}{2}\right)^{(n+1) / 2}-\frac{B_{-}}{r^{n+1}}\left(\frac{2}{\omega}\right)^{(n+1) / 2} \frac{\Gamma\left(\frac{n+1}{2}\right)}{\pi}$.

Matching the above expression with Eq. (19), we find the result

$$
\frac{B_{+}}{B_{-}}=i \frac{\Gamma\left(\frac{n+3}{2}\right)^{2} 2^{n+2}}{\pi\left(\omega r_{H}\right)^{n+2}} .
$$

The reflection coefficient $\mathcal{R}$ for scattering in the gravitational potential of the black hole Eq. (5) is defined as the ratio of the outgoing and incoming amplitude at infinity. To compute this, we expand the far-field solution Eq. (18), in the limit $\omega r \rightarrow \infty$, which yields

$$
\begin{aligned}
R_{\mathrm{FF}}(r) \simeq & \frac{\left(B_{+}-i B_{-}\right)}{\sqrt{2 \pi \omega r^{n+2}}} e^{i[\omega r-(n+2) \pi / 4]} \\
& +\frac{\left(B_{+}+i B_{-}\right)}{\sqrt{2 \pi \omega r^{n+2}}} e^{-i[\omega r-(n+2) \pi / 4]},
\end{aligned}
$$

\footnotetext{
${ }^{2}$ This expansion allows $\omega \lesssim 1 / r_{H}$, the typical emitted energy, when $M \gg M *$, as is required for the reliability of the semiclassical approach.
}

and which, in turn, leads to the following expression for the reflection coefficient:

$$
\mathcal{R}=\frac{\text { outgoing amplitude }}{\text { incoming amplitude }}=\frac{B_{+}-i B_{-}}{B_{+}+i B_{-}},
$$

up to a purely imaginary phase that will drop out when the magnitude of $\mathcal{R}$ will be computed. The absorption probability is then defined as

$$
|\mathcal{A}|^{2}=\left(1-|\mathcal{R}|^{2}\right) \simeq \frac{\pi\left(\omega r_{H}\right)^{n+2}}{2^{n} \Gamma\left(\frac{n+3}{2}\right)^{2}},
$$

where, in the final expression, we have expanded to leading order in $\left(\omega r_{H}\right)$. This is the final result for the $S$-wave greybody factor for scalars in a $(4+n)$-dimensional Schwarzschild black hole background. We may easily check that, for $n=1$, we correctly reproduce the result

$$
|\mathcal{A}|^{2}=\frac{\pi}{2} \omega^{2} r_{H}^{3}=\frac{\omega^{3}}{4 \pi} A_{H},
$$

where $A_{H}=2 \pi^{2} r_{H}^{3}$ is the area of the horizon, presented in Ref. [18].

\section{BULK SCALAR EMISSION FOR $l \geqslant 0$}

We will now generalize the above analysis in the case in which the scalar modes are not spherically symmetric, $l$ $\neq 0$. For readers more interested in our final results, rather than the techniques used to solve the problem, we suggest jumping to Eq. (37) and the following discussion.

We will start with the derivation of the solution in the near-horizon zone. Starting from Eq. (10), and making a change of variable, we may write the scalar field equation in the form (we adopt the method of Ref. [19], see also Ref. [20])

$$
\begin{aligned}
h(1 & -h) \frac{d^{2} R}{d h^{2}}+(1-h) \frac{d R}{d h} \\
& +\left[\frac{(\omega r)^{2}}{(n+1)^{2} h(1-h)}-\frac{l(l+1+n)}{(n+1)^{2}(1-h)}\right] R=0 .
\end{aligned}
$$

Near the horizon, $r \simeq r_{H}$ and the quantity $(\omega r)^{2}$ can be set equal to $\left(\omega r_{H}\right)^{2}$. Then, by using the redefinition $R(h)$ $=h^{\alpha}(1-h)^{\beta} F(h)$, the above equation takes the form of the hypergeometric equation

$$
h(1-h) \frac{d^{2} F}{d h^{2}}+[c-(1+a+b) h] \frac{d F}{d h}-a b F=0,
$$

with $a=b=\alpha+\beta$ and $c=1+2 \alpha$, where 


$$
\begin{aligned}
& \alpha_{ \pm}= \pm \frac{i \omega r_{H}}{n+1}, \\
& \beta_{ \pm}=\frac{1}{2} \pm \frac{1}{n+1} \sqrt{\left(l+\frac{n+1}{2}\right)^{2}-\left(\omega r_{H}\right)^{2}}
\end{aligned}
$$

Equation (27) has as a solution the hypergeometric function $F(a, b, c ; h)$. The criterion for the convergence of the hypergeometric function demands that $\operatorname{Re}(c-a-b)>0$, which forces us to choose $\beta=\beta_{-}$. Then, the general solution of Eq. (26) may be written as [17]

$$
\begin{aligned}
R_{\mathrm{NH}}(h)= & A_{-} h^{\alpha_{ \pm}}(1-h)^{\beta} F(a, b, c ; h)+A_{+} h^{-\alpha_{ \pm}}(1-h)^{\beta} \\
& \times F(a-c+1, b-c+1,2-c ; h) .
\end{aligned}
$$

Expanding the above solution in the limit $r \rightarrow r_{H}$, or $h \rightarrow 0$, and choosing $\alpha=\alpha_{-}$, we obtain the result

$$
\begin{aligned}
R_{\mathrm{NH}} \simeq & \left(\frac{r_{H}}{r}\right)^{\beta(n+1)}\left[A_{-} \exp \left(-i \omega r_{H}^{n+2} y\right)\right. \\
& \left.+A_{+} \exp \left(i \omega r_{H}^{n+2} y\right)\right],
\end{aligned}
$$

which again imposes the condition ${ }^{3} A_{+}=0$.
The derivation of the far-field-zone solution closely follows the analysis performed in the case where $l=0$. The same redefinition of the radial function leads again to a Bessel equation whose general solution is now given by

$$
R_{\mathrm{FF}}(r)=\frac{B_{+}}{r^{(n+1) / 2}} J_{l+(n+1) / 2}(\omega r)+\frac{B_{-}}{r^{(n+1) / 2}} Y_{l+(n+1) / 2}(\omega r) .
$$

Both solutions, near-horizon and far-field, need to be "stretched" and matched in the intermediate region. Expanding first Eq. (31), in the limit $\omega r \ll 1$, gives

$$
\begin{aligned}
R_{\mathrm{FF}}(r) \simeq & \frac{B_{+} r^{l}}{\Gamma\left(l+\frac{n+3}{2}\right)}\left(\frac{\omega}{2}\right)^{l+(n+1) / 2} \\
& -\frac{B_{-}}{r^{l+n+1}}\left(\frac{2}{\omega}\right)^{l+(n+1) / 2} \frac{\Gamma\left(l+\frac{n+1}{2}\right)}{\pi} .
\end{aligned}
$$

The near-horizon solution needs to be "shifted" first and expressed in terms of $1-h$, before being expanded in the limit $r \gg r_{H}$. By using a standard formula [17], we write

$$
\begin{aligned}
R_{\mathrm{NH}}(h)= & A_{-} h^{\alpha}\left[(1-h)^{\beta} \frac{\Gamma(1+2 \alpha) \Gamma(1-2 \beta)}{\Gamma(1+\alpha-\beta)^{2}} F(a, b, a+b-c+1 ; 1-h)\right. \\
& \left.+(1-h)^{1-\beta} \frac{\Gamma(1+2 \alpha) \Gamma(2 \beta-1)}{\Gamma(\alpha+\beta)^{2}} F(c-a, c-b, c-a-b+1 ; 1-h)\right] .
\end{aligned}
$$

We can now expand the above expression in the limit $h \rightarrow 1$ and take

$$
R_{\mathrm{NH}}(h) \simeq A_{-}\left(\frac{r}{r_{H}}\right)^{l} \frac{\Gamma(1+2 \alpha) \Gamma(1-2 \beta)}{\Gamma(1+\alpha-\beta)^{2}}+A_{-}\left(\frac{r_{H}}{r}\right)^{l+n+1} \frac{\Gamma(1+2 \alpha) \Gamma(2 \beta-1)}{\Gamma(\alpha+\beta)^{2}} .
$$

Matching the two solutions Eqs. (32) and (34), we obtain the ratio

$$
\frac{B_{+}}{B_{-}}=-\left(\frac{2}{\omega r_{H}}\right)^{2 l+n+1} \frac{\Gamma\left(l+\frac{n+1}{2}\right)^{2}\left(l+\frac{n+1}{2}\right) \Gamma(1-2 \beta) \Gamma(\alpha+\beta)^{2}}{\pi \Gamma(1+\alpha-\beta)^{2} \Gamma(2 \beta-1)} .
$$

The definition of the reflection coefficient $\mathcal{R}$ is still given by Eq. (23). In turn, the absorption probability can be written, in terms of $B=B_{+} / B_{-}$, as

$$
|\mathcal{A}|^{2}=\left(1-|\mathcal{R}|^{2}\right)=\frac{2 i\left(B^{*}-B\right)}{B B^{*}+i\left(B^{*}-B\right)+1} .
$$

\footnotetext{
${ }^{3}$ Note that the choice $\alpha=\alpha_{+}$would have led again to Eq. (30) with $A_{-} \leftrightarrow A_{+}$, and therefore to the choice $A_{-}=0$. As both values of $\alpha$ appear in the general solution Eq. (29), it is only a matter of choice which one of the two terms will be associated with the incoming mode.
}

Due to the fact that the argument of the $\Gamma$ functions appearing in the expression of $\mathcal{R}$ are nontrivial complex numbers, we cannot write the absorption coefficient in a simple way in the general case. However, the above expression can be further simplified in the limit $\omega r_{H} \ll 1$, in which case $B B^{*}$ $\gg i\left(B^{*}-B\right) \gg 1$, and, therefore, $|\mathcal{A}|^{2}$ may be written as

$$
|\mathcal{A}|^{2}=\frac{4 \pi^{2}}{2^{4 l /(n+1)}}\left(\frac{\omega r_{H}}{2}\right)^{2 l+n+2} \frac{\Gamma\left(1+\frac{l}{n+1}\right)^{2}}{\Gamma\left(\frac{1}{2}+\frac{l}{n+1}\right)^{2} \Gamma\left(l+\frac{n+3}{2}\right)^{2}} .
$$


TABLE I. Absorption probabilities for a $(4+n)$ bulk scalar field.

$$
\begin{array}{lll}
\hline \hline n=2 & l=0 & |\mathcal{A}|^{2} \simeq \frac{4}{9}\left(\omega r_{H}\right)^{4}+\cdots \\
& l=1 & |\mathcal{A}|^{2} \simeq \frac{2^{2 / 3}}{(15)^{2}} \frac{\Gamma(4 / 3)^{2}}{\Gamma(5 / 6)^{2}} \pi\left(\omega r_{H}\right) 6+\cdots \\
& l=2 & |\mathcal{A}|^{2} \simeq \frac{2^{-2 / 3}}{(105)^{2}} \frac{\Gamma(5 / 3)^{2}}{\Gamma(7 / 6)^{2}} \pi\left(\omega r_{H}\right)^{8}+\cdots \\
n=4 & l=0 & |\mathcal{A}|^{2} \simeq \frac{4}{(15)^{2}}\left(\omega r_{H}\right)^{6}+\cdots \\
& l=1 & |\mathcal{A}|^{2} \simeq \frac{2^{6 / 5}}{(105)^{2}} \frac{\Gamma(6 / 5)^{2}}{\Gamma(7 / 10)^{2}} \pi\left(\omega r_{H}\right)^{8}+\cdots \\
n=6 & |\mathcal{A}|^{2} \simeq \frac{2^{2 / 5}}{(105)^{2} 3^{4}} \frac{\Gamma(7 / 5)^{2}}{\Gamma(9 / 10)^{2}} \pi\left(\omega r_{H}\right)^{10}+\cdots \\
& l=0 & |\mathcal{A}|^{2} \simeq \frac{4}{(105)^{2}}\left(\omega r_{H}\right)^{8}+\cdots \\
& l=1 & |\mathcal{A}|^{2} \simeq \frac{22^{3 / 7}}{(105)^{2} 3^{4}} \frac{\Gamma(8 / 7)^{2}}{\Gamma(9 / 14)^{2}} \pi\left(\omega r_{H}\right)^{10}+\cdots \\
& \left.l \mathcal{A}\right|^{2} \simeq \frac{2^{6 / 7}}{(1155)^{2} 3^{4}} \frac{\Gamma(9 / 7)^{2}}{\Gamma(11 / 14)^{2}} \pi\left(\omega r_{H}\right)^{12}+\cdots \\
& l=2
\end{array}
$$

This is our major result for the case of bulk scalar fields. Equation (37) nicely displays the leading functional dependence of the greybody factor on $\omega r_{H}$ for varying partial wave, $l$, and number of extra dimensions, $n$. For the case of $l=0$, this may be further evaluated to give

$$
|\mathcal{A}|_{l=0}^{2}=\left(\frac{\omega r_{H}}{2}\right)^{n+2} \frac{4 \pi}{\Gamma[(n+3) / 2]^{2}},
$$

in complete agreement with the result, Eq. (24), of our earlier analysis in the case $l=0$. In fact, for an $s$-wave massless bulk scalar, the absorption probability $|\mathcal{A}|^{2}$ has the exact form that allows the greybody factor $\sigma(\omega)$ to reduce to the horizon area of the black hole in agreement with previous work [21]. The numerical values of the results for the cases $l=0,1,2$ for $n=2,4$, and 6 are shown in Table I.

If we fix the number of extra dimensions and vary only the angular momentum number, the absorption probability decreases as $l$ increases. This decrease is caused by the fact that the power of the expansion parameter $\left(\omega r_{H}\right)^{2 l+n+2}$, in the leading term, increases with $l$. Since $\left(\omega r_{H}\right) \ll 1$, this means that $|\mathcal{A}|^{2}$ becomes more and more suppressed as $l$ increases. The numerical coefficient in front of the leading term also decreases with $l$ (see Table I). The same behavior is observed if we fix instead $l$ and vary $n$.

\section{BRANE-LOCALIZED SCALAR EMISSION FOR $l \geqslant 0$}

We now turn to the case in which the scalar field is confined on a four-dimensional brane embedded in a $(4+d)$-dimensional Schwarzschild spacetime. The scalar field propagates in a four-dimensional background whose metric tensor is given by the induced metric at the location of the brane. The induced metric follows from the $(4+d)$-dimensional one by fixing the values of the extra angular coordinates, $\theta_{n}=\pi / 2$ for $n \geqslant 2$, and it may be written as

$$
d s^{2}=-h(r) d t^{2}+h(r)^{-1} d r^{2}+r^{2}\left(d \theta^{2}+\sin ^{2} \theta d \varphi^{2}\right),
$$

where $h(r)$ is still given by Eq. (6). The scalar field equation may be separated in the same way,

$$
\phi(t, r, \theta, \varphi)=e^{-i \omega t} R_{\omega l}(r) Y_{l}(\Omega),
$$

where $Y_{l}(\Omega)$ are now the usual three-dimensional spherical harmonic functions. The above ansatz allows us to write the equation for the radial part as

$$
\frac{h(r)}{r^{2}} \frac{d}{d r}\left[h(r) r^{2} \frac{d R}{d r}\right]+\left[\omega^{2}-\frac{h(r)}{r^{2}} l(l+1)\right] R=0 .
$$

The presence of the metric function $h(r)$ makes once again the derivation of the general solution extremely difficult. We will follow the same method as in the previous section and compute the solution in the two radial domains, near-horizon and far-field, which will then be "stretched" and matched in the intermediate region.

Having become familiar with the analysis, we will proceed to derive directly the solution in the general case $l$ $\geqslant 0$. We start with the solution in the "near-horizon" region. In terms of $h$, the radial differential equation now takes the form

$$
\begin{aligned}
h(1 & -h) \frac{d^{2} R}{d h^{2}}+\left[1-\frac{(2 n+1)}{(n+1)} h\right] \frac{d R}{d h} \\
& +\left[\frac{\left(\omega r_{H}\right)^{2}}{(n+1)^{2} h(1-h)}-\frac{l(l+1)}{(n+1)^{2}(1-h)}\right] R=0 .
\end{aligned}
$$

By using the same redefinition $R(h)=h^{\alpha}(1-h)^{\beta} F(h)$, the above equation assumes the standard form of a hypergeometric equation with indices $a=\alpha+\beta+[n /(n+1)], b=\alpha+\beta$, and $c=1+2 \alpha$, where

$$
\begin{aligned}
& \alpha_{ \pm}= \pm \frac{i \omega r_{H}}{n+1} \\
& \beta_{ \pm}=\frac{1}{2(n+1)}\left[1 \pm \sqrt{(2 l+1)^{2}-4\left(\omega r_{H}\right)^{2}}\right]
\end{aligned}
$$

The criterion for the convergence of the hypergeometric function, $\operatorname{Re}(c-a-b)>0$, demands again $\beta=\beta_{-}$. The general solution of Eq. (42) has again the form of Eq. (29). Expanding near the horizon and imposing the condition that only incoming waves exist near $r \simeq r_{H}$, we find that $A_{+}=0$ for $\alpha=\alpha_{-}$. At this point, we can also "shift" the solution and write it in terms of $1-h$, in the same way as in the previous section. If we finally expand for $r \gg r_{H}$, or equivalently $h \rightarrow 1$, we obtain the solution 


$$
\begin{aligned}
R_{\mathrm{NH}}(h) \simeq A_{-}\left(\frac{r}{r_{H}}\right) & \frac{\Gamma(1+2 \alpha) \Gamma\left(1-2 \beta-\frac{n}{n+1}\right)}{\Gamma\left(1+\alpha-\beta-\frac{n}{n+1}\right) \Gamma(1+\alpha-\beta)} \\
& +A_{-}\left(\frac{r_{H}}{r}\right)^{l+1} \frac{\Gamma(1+2 \alpha) \Gamma\left(2 \beta+\frac{n}{n+1}-1\right)}{\Gamma\left(\alpha+\beta+\frac{n}{n+1}\right) \Gamma(\alpha+\beta)}
\end{aligned}
$$

The far-field-zone solution can be easily found to be given in terms of the Bessel functions $J_{l+1 / 2}(\omega r)$ and $Y_{l+1 / 2}(\omega r)$. Expanding the general solution in the limit $\omega r \ll 1$ finally gives

$$
R_{\mathrm{FF}}(r) \simeq \frac{B_{+} r^{l}}{\Gamma\left(l+\frac{3}{2}\right)}\left(\frac{\omega}{2}\right)^{l+1 / 2}-\frac{B_{-}}{r^{l+1}}\left(\frac{2}{\omega}\right)^{l+1 / 2} \frac{\Gamma\left(l+\frac{1}{2}\right)}{\pi}
$$

Matching the two asymptotic solutions, we obtain the ratio

$$
\frac{B_{+}}{B_{-}}=-\left(\frac{2}{\omega r_{H}}\right)^{2 l+1} \frac{\Gamma\left(l+\frac{1}{2}\right)^{2}\left(l+\frac{1}{2}\right) \Gamma\left(1-2 \beta-\frac{n}{n+1}\right) \Gamma(\alpha+\beta) \Gamma\left(\alpha+\beta+\frac{n}{n+1}\right)}{\pi \Gamma(1+\alpha-\beta) \Gamma\left(1+\alpha-\beta-\frac{n}{n+1}\right) \Gamma\left(2 \beta+\frac{n}{n+1}-1\right)},
$$

which can be used to determine the absorption coefficient according to Eq. (36). We may, however, obtain a simplified expression, in the limit $\omega r_{H} \ll 1$, which reads

$$
|\mathcal{A}|^{2}=\frac{16 \pi}{(n+1)^{2}}\left(\frac{\omega r_{H}}{2}\right)^{2 l+2} \frac{\Gamma\left(\frac{l+1}{n+1}\right)^{2} \Gamma\left(1+\frac{l}{n+1}\right)^{2}}{\Gamma\left(\frac{1}{2}+l\right)^{2} \Gamma\left(1+\frac{2 l+1}{n+1}\right)^{2}} .
$$

The expressions of the absorption coefficient $|\mathcal{A}|^{2}$ for the

TABLE II. Absorption probabilities for a (4D) brane scalar field.

\begin{tabular}{lll}
\hline \hline$n=2$ & $l=0$ & $|\mathcal{A}|^{2} \simeq 4\left(\omega r_{H}\right)^{2}+\cdots$ \\
& $l=1$ & $|\mathcal{A}| \simeq \frac{16 \pi^{2}}{243}\left(\omega r_{H}\right)^{4}+\cdots$ \\
& $l=2$ & $|\mathcal{A}|^{2} \simeq \frac{4}{(15)^{2}}\left(\omega r_{H}\right)^{6}+\cdots$ \\
$n=4$ & $l=0$ & $|\mathcal{A}|^{2} \simeq 4\left(\omega r_{H}\right)^{2}+\cdots$ \\
& $l=1$ & $|\mathcal{A}|^{2} \simeq \frac{4}{25} \frac{\Gamma(2 / 5)^{2} \Gamma(6 / 5)^{2}}{\Gamma(8 / 5)}\left(\omega r_{H}\right)^{4}+\cdots$ \\
& $l=2$ & $|\mathcal{A}|^{2} \simeq \frac{4 \Gamma(3 / 5)^{2} \Gamma(7 / 5)^{2}}{(15)^{2}}\left(\omega r_{H}\right)^{6}+\cdots$ \\
$n=6$ & $l=0$ & $|\mathcal{A}|^{2} \simeq 4\left(\omega r_{H}\right)^{2}+\cdots$ \\
& $l=1$ & $\frac{4(2 / 7)^{2} \Gamma(8 / 7)^{2}}{49}\left(\omega r_{H}\right)^{4}+\cdots$ \\
& & $|\mathcal{A}|^{2} \simeq \frac{4}{(21)^{2}} \frac{\Gamma(3 / 7)^{2} \Gamma(9 / 7)^{2}}{\Gamma(12 / 7)^{2}}\left(\omega r_{H}\right)^{6}+\cdots$
\end{tabular}

values $n=2,4$, and 6 of the number of extra dimensions, and $l=0,1,2$ of the angular momentum number, are shown in Table II.

Finally, employing the relation between the absorption probability and the greybody factor, Eq. (13), leads to

$$
\sigma_{l}(\omega)=\frac{4 \pi^{2}(2 l+1)}{(n+1)^{2}} \frac{\Gamma\left(\frac{l+1}{n+1}\right)^{2} \Gamma\left(1+\frac{l}{n+1}\right)^{2}}{\Gamma\left(\frac{1}{2}+l\right)^{2} \Gamma\left(1+\frac{2 l+1}{n+1}\right)^{2}}\left(\frac{\omega r_{H}}{2}\right)^{2 l} r_{H}^{2} .
$$

In this equation, we have set $n=0$ in Eq. (13) as the partial waves are purely confined to the three-spatial-dimensional brane, the only dependence on $n$ being in $|\mathcal{A}|^{2}$.

Keeping $n$ fixed and varying $l$, we see that the absorption probability decreases once again as $l$ increases (see Table II): the dominant term becomes more and more suppressed by extra powers of $\left(\omega r_{H}\right)$ and its numerical coefficient also decreases (the introduction of the multiplicity of states with the same angular momentum number $l$ does not change this behavior). If we fix instead $l$ and vary $n$, a radically different behavior from the one observed in the case with a bulk scalar field emerges, namely the leading term, in the expansion of $|\mathcal{A}|^{2}$ in powers of $\left(\omega r_{H}\right)$, remains the same, since it is $n$-independent, while its coefficient increases as $n$ increases. In other words, for a given partial wave, the absorption probability, and therefore the greybody factor, increases as the number of extra dimensions being projected on the 3-brane also increases.

\section{CONCLUSIONS}

In this paper, we have studied the problem of scalar emission in a spherically symmetric $D$-dimensional Schwarzschild black hole background. The cases of the emission of a 
$(4+n)$-dimensional bulk scalar field and of a fourdimensional brane-localized scalar field were studied separately and the greybody factor was determined in each case. This quantity causes the spectrum of Hawking radiation to deviate from the blackbody spectrum as it strongly depends on the energy of the particle mode emitted. Moreover, it encodes information about the gravitational background and thus on the number of extra dimensions that might exist [both in the case where the scalar field is free to propagate in the $(4+n)$-dimensional bulk or when the field "feels" the existence of extra dimension only through the induced metric on our 3-brane].

The differential equation for the radial part of the scalar field can be solved by an approximation method valid in leading order in $\left(\omega r_{H}\right)$ : the solution of this equation was found in the "near-horizon" and "far-field" region and was subsequently "stretched" and matched in an intermediate regime. This matching allows us to determine the absorption coefficient for scattering in the black hole background, which then leads to the greybody factor, $\sigma(\omega)$, according to Eq. (13).

We first focused on the case of a bulk scalar field propagating in a $(4+n)$-dimensional Schwarzschild black hole background. The general form for the amplitude probability was determined and an analytical, simplified version was also presented that allowed us to display the leading functional dependence on the expansion parameter $\left(\omega r_{H}\right)$ in terms of the number $n$ of extra dimension and the angular momentum number $l$. Our results in this case are presented in Eq. (37) and in Table I.

The most phenomenologically interesting case is the one of a scalar field that is confined on a 3-brane and propagates in the induced spacetime background of a black hole (which is necessarily higher-dimensional). The functional form of the resulting greybody factor depends only on the angular momentum number, $l$, through $\left(\omega r_{H}\right)^{2 l+2}$. The dependence on the number of extra dimensions is entirely contained within the coefficient of this leading term. Our primary results are given in Eq. (48) and in Table II.

It is tempting to compare the results derived in the case of a brane-localized scalar field (which nevertheless is part of a higher-dimensional manifold) with those valid in the case where a purely four-dimensional scalar field propagates in a Schwarzschild black hole background. Both cases lead to the same value of $|\mathcal{A}|^{2}$ for an $s$ wave and, thus, no distinction can be made between the two backgrounds. However, for higher partial waves, the value of the absorption probability
TABLE III. Absorption probabilities for a (4D) scalar field.

\begin{tabular}{ll}
\hline \hline$l=0$ & $|\mathcal{A}|^{2} \simeq 4\left(\omega r_{H}\right)^{2}+\cdots$ \\
$l=1$ & $|\mathcal{A}|^{2} \simeq \frac{1}{9}\left(\omega r_{H}\right)^{4}+\cdots$ \\
$l=2$ & $|\mathcal{A}|^{2} \simeq \frac{1}{(45)^{2}}\left(\omega r_{H}\right)^{6}+\cdots$ \\
\hline
\end{tabular}

in the case of a brane scalar field is always larger than the one for a purely 4D field, a fact which in principle can be used to distinguish between the two cases.

In a companion paper [22], we employ the techniques developed in the current paper to derive the greybody factors for higher-spin fields localized on a brane. This allows us to discuss the physics and phenomenology of black hole decay, as might be observed at the LHC.

\section{APPENDIX}

For completeness, we present here some of the calculation of scalar emission in the case in which the scalar field propagates in a purely four-dimensional Schwarzschild background without the assumption of the presence of extra dimensions. The expression of the absorption coefficient $|\mathcal{A}|^{2}$ can be easily found by first putting $n=0$ (the number of projected extra dimensions on the four-dimensional plane) in the result for the ratio $B_{+} / B_{-}$. Then, Eq. (46) becomes

$$
\frac{B_{+}}{B_{-}}=-\left(\frac{2}{\omega r_{H}}\right)^{2 l+1} \frac{\Gamma\left(l+\frac{1}{2}\right)^{2}\left(l+\frac{1}{2}\right) \Gamma(1-2 \beta) \Gamma(\alpha+\beta)^{2}}{\pi \Gamma(1+\alpha-\beta)^{2} \Gamma(2 \beta-1)},
$$

where now

$$
\alpha=-i \omega r_{H}, \quad \beta=\frac{1}{2}\left[1-\sqrt{(2 l+1)^{2}-4\left(\omega r_{H}\right)^{2}}\right] .
$$

By using then Eq. (36), we obtain the result for $|\mathcal{A}|^{2}$ which, in its simplified form, reads

$$
|\mathcal{A}|^{2}=16 \pi\left(\frac{\omega r_{H}}{2}\right)^{2 l+2} \frac{\Gamma(l+1)^{4}}{\Gamma\left[\frac{1}{2}+l\right]^{2} \Gamma[2 l+2]^{2}} .
$$

Note that the same results follow by putting $n=0$ in all the expressions of Sec. II A, as expected.

We display the results for the absorption coefficient in the pure four-dimensional case and for the values $l=0,1,2$ of the angular momentum number in Table III.
[1] N. Arkani-Hamed, S. Dimopoulos, and G. R. Dvali, Phys. Lett. B 429, 263 (1998); I. Antoniadis, N. Arkani-Hamed, S. Dimopoulos, and G. R. Dvali, ibid. 436, 257 (1998); N. ArkaniHamed, S. Dimopoulos, and G. R. Dvali, Phys. Rev. D 59, 086004 (1999).

[2] K. Akama, Lect. Notes Phys. 176, 267 (1982); V. A. Rubakov and M. E. Shaposhnikov, Phys. Lett. 125B, 139 (1983); 125B, 136 (1983); M. Visser, ibid. 159B, 22 (1985); I. Antoniadis,
Phys. Lett. B 246, 377 (1990); I. Antoniadis, K. Benakli, and M. Quiros, ibid. 331, 313 (1994); J. D. Lykken, Phys. Rev. D 54, 3693 (1996).

[3] L. Randall and R. Sundrum, Phys. Rev. Lett. 83, 3370 (1999); 83, 4690 (1999); I. Antoniadis, K. Benakli, and M. Quiros, Phys. Lett. B 460, 176 (1999); N. Arkani-Hamed, S. Dimopoulos, and J. March-Russell, Phys. Rev. D 63, 064020 (2001); R. Sundrum, ibid. 59, 085009 (1999); G. F. Giudice, R. Rattazzi, 
and J. D. Wells, Nucl. Phys. B544, 3 (1999); E. A. Mirabelli, M. Perelstein, and M. E. Peskin, Phys. Rev. Lett. 82, 2236 (1999); J. L. Hewett, ibid. 82, 4765 (1999); T. Han, J. D. Lykken, and R. J. Zhang, Phys. Rev. D 59, 105006 (1999); N. Kaloper, J. March-Russell, G. D. Starkman, and M. Trodden, Phys. Rev. Lett. 85, 928 (2000); S. Cullen and M. Perelstein, ibid. 83, 268 (1999); S. Hannestad and G. Raffelt, ibid. 87, 051301 (2001); L. J. Hall and D. R. Smith, Phys. Rev. D 60, 085008 (1999); N. Arkani-Hamed, S. Dimopoulos, G. R. Dvali, and J. March-Russell, ibid. 65, 024032 (2002); K. R. Dienes, E. Dudas, and T. Gherghetta, Nucl. Phys. B557, 25 (1999); B537, 47 (1999); N. Arkani-Hamed, S. Dimopoulos, N. Kaloper, and J. March-Russell, ibid. B567, 189 (2000); S. Cullen, M. Perelstein, and M. E. Peskin, Phys. Rev. D 62, 055012 (2000); P. Kanti and K. A. Olive, ibid. 60, 043502 (1999); Phys. Lett. B 464, 192 (1999).

[4] P. C. Argyres, S. Dimopoulos, and J. March-Russell, Phys. Lett. B 441, 96 (1998).

[5] R. C. Myers and M. J. Perry, Ann. Phys. (N.Y.) 172, 304 (1986).

[6] S. B. Giddings and S. Thomas, Phys. Rev. D 65, 056010 (2002)

[7] S. Dimopoulos and G. Landsberg, Phys. Rev. Lett. 87, 161602 (2001).

[8] S. Dimopoulos and R. Emparan, Phys. Lett. B 526, 393 (2002); G. F. Giudice, R. Rattazzi, and J. D. Wells, Nucl. Phys. B630, 293 (2002); D. M. Eardley and S. B. Giddings, gr-qc/0201034.

[9] M. B. Voloshin, Phys. Lett. B 518, 137 (2001); 524, 376 (2002).

[10] J. L. Feng and A. D. Shapere, Phys. Rev. Lett. 88, 021303 (2002); R. Emparan, M. Masip, and R. Rattazzi, Phys. Rev. D 65, 064023 (2002); L. A. Anchordoqui, J. L. Feng, H. Goldberg, and A. D. Shapere, ibid. 65, 124027 (2002); Y. Uehara, hep-ph/0110382; J. Alvarez-Muniz, J. L. Feng, F. Halzen, T. Han, and D. Hooper, Phys. Rev. D 65, 124015 (2002); A.
Ringwald and H. Tu, Phys. Lett. B 525, 135 (2002); M. Kowalski, A. Ringwald, and H. Tu, ibid. 529, 1 (2002); D. Kazanas and A. Nicolaidis, hep-ph/0109247; P. Jain, S. Kar, S. Panda, and J. P. Ralston, hep-ph/0201232.

[11] U. Baur et al., in Proceedings of the APS/DPF/DPB Summer Study on the Future of Particle Physics (Snowmass, 2001), edited by R. Davidson and C. Quigg, hep-ph/0201227; K. Cheung, Phys. Rev. Lett. 88, 221602 (2002); P. Kanti and K. Tamvakis, Phys. Rev. D 65, 084010 (2002); S. Hofmann et al., hep-ph/0111052; G. Pasztor and T. G. Rizzo, in Proceedings of the APS/DPF/DPB Summer Study on the Future of Particle Physics (Snowmass, 2001), edited by R. Davidson and C. Quigg, hep-ph/0112054; G. Landsberg, Phys. Rev. Lett. 88, 181801 (2002); M. Bleicher, S. Hofmann, S. Hossenfelder, and H. Stocker, hep-ph/0112186; E. J. Ahn, M. Cavaglia, and A. V. Olinto, hep-th/0201042; S. N. Solodukhin, Phys. Lett. B 533, 153 (2002); S. C. Park and H. S. Song, hep-ph/0111069; R. Casadio and B. Harms, hep-th/0110255; S. Hossenfelder, S. Hofmann, M. Bleicher, and H. Stocker, hep-ph/0109085; T. G. Rizzo, J. High Energy Phys. 02, 011 (2002).

[12] R. Emparan, G. T. Horowitz, and R. C. Myers, Phys. Rev. Lett. 85, 499 (2000).

[13] S. W. Hawking, Commun. Math. Phys. 43, 199 (1975).

[14] J. Preskill, hep-th/9209058.

[15] C. Muller, Lecture Notes in Mathematics: Spherical Harmonics (Springer-Verlag, Berlin, 1966).

[16] S. S. Gubser, I. R. Klebanov, and A. A. Tseytlin, Nucl. Phys. B499, 217 (1997).

[17] M. Abramowitz and I. Stegun, Handbook of Mathematical Functions (Academic, New York, 1966).

[18] S. R. Das and S. D. Mathur, Nucl. Phys. B478, 561 (1996).

[19] J. Maldacena and A. Strominger, Phys. Rev. D 55, 861 (1997).

[20] R. Emparan, Nucl. Phys. B516, 297 (1998).

[21] S. R. Das, G. W. Gibbons, and S. D. Mathur, Phys. Rev. Lett. 78, 417 (1997).

[22] P. Kanti and J. March-Russell, work in progress. 